\title{
Dynamic Cerebral Autoregulation Is Transiently Impaired for One Week after Large-Vessel Acute Ischemic Stroke
}

\author{
Nils H. Petersen ${ }^{a}$ Santiago Ortega-Gutierrez ${ }^{a} \quad$ Andrés Reccius $^{b}$ \\ Arjun Masurkar $^{\mathrm{a}} \quad$ Amy Huang $^{\mathrm{a}}$ Randolph S. Marshall ${ }^{\mathrm{a}}$ \\ ${ }^{a}$ Department of Neurology, Columbia University, New York, N.Y., USA; ${ }^{b}$ Clinica Alemana, Universidad del \\ Desarrollocation, Santiago, Chile
}

\section{Key Words \\ Dynamic cerebral autoregulation · Stroke - Transcranial \\ Doppler $\cdot$ Transfer function analysis}

\begin{abstract}
Background: Dynamic cerebral autoregulation (DCA) is the continuous counterregulation of cerebral blood flow to fluctuations in blood pressure. DCA can become impaired after acute stroke, but it remains unclear to what extent and over what interval this occurs. Methods: We included 28 patients (NIHSS $=12 \pm 6.5$, age $=68.4 \pm 17.1,16 \mathrm{~F}$ ) with acute large-vessel ischemic stroke in the middle cerebral artery territory and 29 healthy controls (mean age $54.9 \pm 9,16 \mathrm{~F}$ ). DCA was assessed by simultaneous measurement of blood pressure together with blood flow velocities using finger plethysmography/arterial catheter and transcranial Doppler over three 10-minute recordings on days 0-2, 3-6 and $\geq 7$ days after stroke. Transfer function analysis was applied to calculate average phase shift (PS) in the low frequency range $(0.06-0.12 \mathrm{~Hz})$. Less PS indicated poorer autoregulation. The affected side was compared with the unaffected side and controls. Univariate comparisons of data were performed using $t$ tests at single time points, and generalized estimating equations with an exchangeable correlation ma-
\end{abstract}

trix to examine the change in PS over time. Results: At mean $1.3 \pm 0.5$ days after stroke the average PS in the affected hemisphere was $29.6 \pm 10.5$ vs. $42.5 \pm 13$ degrees in the unaffected hemisphere $(p=0.004)$. At $4.1 \pm 1$ days, the PS in affected and unaffected hemisphere was $23.2 \pm 19.1$ vs. $41.7 \pm 18.5$ degrees, respectively $(p=0.003)$. At mean $9.75 \pm$ 2.2 days stroke there was no difference between the affected and the unaffected hemisphere $(53.2 \pm 28.2$ vs. $50.7 \pm$ 29.2 degrees, $p=0.69$ ). Control subjects had an average PS $=47.9 \pm 16.8$, significantly different from patients' affected hemisphere at the first two measurements $(p=$ $0.001)$, but not the third ( $p=0.37)$. The PS in controls remained unchanged on repeat testing after an average 19.1 days $(48.4 \pm 17.1, p=0.61)$. Using the last recording as the reference, the average PS in the affected hemisphere was $-23.54(-44.1,-3)$ degrees lower on recording one $(p=$ $0.025)$, and $-31.6(-56.1,-7.1)$ degrees lower on recording two $(p<0.011)$. Changes in the unaffected hemisphere over time were nonsignificant. Discussion: These data suggest that dynamic cerebral autoregulation is impaired in the affected hemisphere throughout the first week after largevessel ischemic stroke, and then normalizes by week two. These findings may have important implications for acute blood pressure management after stroke.

(c) 2015 S. Karger AG, Basel

\section{KARGER 125}

(c) 2015 S. Karger AG, Base

$1015-9770 / 15 / 0392-0144 \$ 39.50 / 0$ 


\section{Introduction}

Under normal circumstances cerebral blood flow is maintained over a wide range of systemic blood pressures, a phenomenon known as cerebral autoregulation (CA). This mechanism ensures that the cerebral blood flow matches the brain's metabolic demands and protects it from hypo- or hyperperfusion. The active response of cerebral blood vessels to spontaneous or induced blood pressure fluctuations is also referred to as dynamic cerebral autoregulation (DCA) $[1,2]$. DCA may become impaired after ischemic stroke [3-6]; in that setting CBF is likely to depend on cerebral perfusion pressure (CPP), rendering the brain at risk for secondary injury such as further ischemia, hemorrhagic transformation and edema formation. Blood pressure is often elevated early after ischemic stroke $[7,8]$ and its management in the setting of acute stroke remains unclear. Both high and low blood pressures during the acute stroke period have been associated with poor outcome [9-11]. Given the lack of definitive data, clinical guidelines are based on theoretical assumptions about autoregulation in the ischemic penumbra, and recommend against the administration of antihypertensive agents unless the blood pressure exceeds values $>220 / 120$ [12]. However, the temporal course of autoregulatory disturbance remains unknown. Transcranial Doppler ultrasonography (TCD) combined with servo-controlled finger photoplethysmography (Finapres) has allowed continuous, non-invasive assessment of dynamic cerebral autoregulation from spontaneous blood pressure fluctuations $[13,14]$. This approach eliminates the need for potentially harmful induced blood pressure or vasodilatory interventions and allows noninvasive serial assessments of autoregulatory function in the acute stroke period. This study sets out to describe the specific temporal course of cerebral autoregulation in patients with acute ischemic stroke.

\section{Methods}

\section{Subjects and Measurements}

Patients with acute ischemic stroke admitted to the Stroke unit or Neurological ICU at Columbia University Medical Center were eligible for the study. DCA measurements were performed on days $0-2,3-6$ and $\geq 7$ days after stroke. Inclusion criteria were MCA territory ischemic stroke, first measurement within $48 \mathrm{~h}$ of onset and age greater than 18. Patients were excluded from the study if they had prior clinical stroke, extracranial stenosis greater than $70 \%$, prior $\mathrm{SAH}$ or $\mathrm{ICH}$, an inadequate acoustic window, or treatment with vasopressors. Consent was obtained from the patient or the surrogate decision maker prior to enrollment. All patients were provided with detailed written information regarding the intent and methods of the study. Approval for the study was obtained from the Institutional Review Board at Columbia University Medical Center.

Reference autoregulation values were obtained from 29 control subjects without cerebrovascular disease; 8 of them had a repeat study after 20 days.

DCA measurements were performed in the supine position with slight elevation of the upper body while the subjects were breathing spontaneously. Cerebral blood flow velocities were assessed using transcranial Doppler (DWL-Multidop-X, Sipplingen, Germany). Proximal MCA patency was a prerequisite for the Doppler monitoring. The proximal middle cerebral artery was insonated through the temporal window with a $2 \mathrm{MHz}$ probe attached to a head frame, at a depth of insonation of 45-60 mm. Blood pressure was recorded simultaneously using intraarterial catheter or servo-controlled finger plethysmography (Finometer Pro, Amsterdam, the Netherlands). The appropriate finger cuff (size: small, medium or large) was placed on the middle phalanx of the left or right middle finger. After establishing a stable recording, the intermittently occurring calibration procedure was turned off. Data was recorded for $10 \mathrm{~min}$. All analog signals were digitized and stored for editing and offline analysis. Data sampling frequency was $100 \mathrm{~Hz}$.

\section{Data Analysis}

The editing process included temporal synchronization of the blood pressure and blood flow velocity wave forms using ICUpilot software (Dipylon Medical, Solna, Sweden). This was followed by visual inspection and removal of all major artifacts. The data was then analyzed using Matlab (MathWorks, Natick, Mass., USA) with an in-house written program. The relationship between changes in arterial pressure and CBFV was assessed with transfer function analysis.

\section{Transfer Function Analysis (TFA)}

Because the data being acquired takes the form of 2 repetitive oscillatory patterns - arterial blood pressure (ABP) and cerebral blood flow velocity (CBFV) - their spatial relationship can be best characterized using TFA. This method quantifies the extent to which the input signal (ABP) is reflected in the output signal (CBFV), thus quantifying the efficacy of the autoregulatory system [15].

The transfer function $\mathrm{H}(\mathrm{f})$ relating each $\mathrm{CBFV}$ to $\mathrm{ABP}$ was approximated by assuming linearity and time invariance. Estimation of spectra and transfer function was based the method described by Welch and has been outlined in more detail previously $[16,17]$.

In brief, arterial blood pressure ABP and CBFV from the left and right middle cerebral arteries were normalized with respect to the mean prior to frequency spectrum analysis. The Fast Fourier Transform (FFT) algorithm was used to calculate the auto-spectra and the cross spectrum of the two signals (ABP and CBFV). Spectra were calculated using individual segments $1 / 6$ th the total length of the time series with $50 \%$ overlap. Smoothing was accomplished by employing a Hanning window.

The coherence, gain $|H(f)|$ and phase $\Phi(f)$ of the system were calculated. Coherence significance criterion $\left(\gamma_{\mathrm{min}}\right)$, above which coherence differs significantly from 0 , was derived from the degrees of freedom $v$ of the spectral estimate at a significance level $a$ of $0.05[14,18]$. The degrees of freedom are dependent on the de- 
tails of the spectral estimation method and in our case resulted in a coherence significance level, $\gamma_{\min }$ equal to 0.5372 . Phase shift and gain were calculated by averaging the values of all valid bins in the low-frequency range $(0.06-0.12 \mathrm{~Hz}$; each bin is a frequency value at $0.01 \mathrm{~Hz}$ increments) where coherence was $>0.53$.

The resulting phase shift describes to what extent oscillations in CBFV lead those in ABP and can be interpreted as active early counter-regulation. Less phase shift reflects increased latency in cerebral vasomotion and thus poorer autoregulation [19]. Supplemental figure 1 shows an illustration of the concept of TFA.

\section{Statistical Analysis}

Continuous variables were tested for normality using the Kolmogorov-Smirnov test. Data were reported as means and standard deviation (SD) or median, 25th and 75th percentile. Univariate comparisons at single time points were performed using paired $t$ tests. Generalized estimating equations (GEE) with an exchangeable correlation matrix were used to examine the change in phase shift (PS) over time. GEE is an analysis method for longitudinal data when responses are correlated. It accounts for within-subject or within-cluster correlations with a range of correlations structures, and allows for missing data and irregularly timed or mistimed measurements. A p value of less than 0.05 was considered statistically significant.

\section{Results}

From May 2011 to March 2012 we recruited 28 patients for the study and performed a total of 56 measurements. Twenty-nine age-matched subjects without cerebrovascular disease served as controls. Demographics and baseline characteristics are given in table 1 .

At the first two measurements, recordings from four patients could not be obtained due to technical difficulties or the patient's inability to cooperate with the exam. On the third measurement, recordings were performed on 8 patients; the remainder had already been discharged from the hospital. Overall, we found a significant decrease in phase shift in the affected hemisphere at the first two measurements followed by normalization at the third measurement. Figure 1a-c shows the average phase shift for the affected and unaffected side at the three time points. The first measurement was obtained $1.3 \pm 0.5$ days after the stroke. The average PS in the affected hemisphere was $29.6 \pm 10.5$ degrees vs. $42.5 \pm 13$ degrees in the unaffected hemisphere $(\mathrm{p}=0.004)$. At the second measurement ( $4.1 \pm 1$ days) the PS in the affected and the unaffected hemisphere was $23.2 \pm 19.1$ vs. $41.7 \pm 18.5$ degrees, respectively $(\mathrm{p}=0.003)$. At $9.75 \pm 2.2$ days after the stroke there was no difference between the affected and the unaffected hemisphere $(53.2 \pm 28.2$ vs. $50.7 \pm 29.2$ degrees, $\mathrm{p}=0.69$ ). The results of the hemodynamic and autoregulatory parameters are given in table 2 . Control
Table 1. Demographics and baseline characteristics

\begin{tabular}{lcl}
\hline & $\begin{array}{c}\text { Patients } \\
(\mathrm{n}=28)\end{array}$ & $\begin{array}{l}\text { Controls } \\
(\mathrm{n}=29)\end{array}$ \\
\hline Age, years (mean \pm SD) & $68.4 \pm 17.1$ & $54.9 \pm 9$ \\
NIHSS (mean \pm SD) & $12 \pm 6.5$ & NA \\
Stroke size, cc (mean \pm SD) & $39.7 \pm 50.6$ & NA \\
Gender, n (\%) & $16(57.1)$ & $16(55.2)$ \\
$\quad$ Female & $12(42.9)$ & $13(44.8)$ \\
Male & $6(21.4)$ & $17(58.6)$ \\
Ethnicity, n (\%) & $5(17.9)$ & $2(6.9)$ \\
White & $11(39.3)$ & $5(17.2)$ \\
Black & $0(0)$ & $2(6.9)$ \\
Hispanic & $6(21.5)$ & $3(10.3)$ \\
Asian & & \\
Other & $16(57.1)$ & \\
Side of stroke, $\mathrm{n}(\%)$ & $12(42.9)$ & \\
Left & & \\
Right & $10(35.7)$ & \\
Stroke etiology, n $(\%)$ & $9(32.1)$ & \\
CE & $3(10.7)$ & \\
LAA & $6(21.4)$ & \\
SOE & & \\
SUE & & \\
\hline
\end{tabular}

Stroke etiology according to TOAST criteria. $\mathrm{CE}=$ Cardio-embolism; LAA = large artery atherosclerosis; $\mathrm{SOE}=$ stroke of other etiology; SUE = stroke of undetermined etiology.

subjects had an average PS $=47.9 \pm 16.8$, significantly different from the affected hemisphere at the first two measurements $(\mathrm{p}<0.001)$, but not the third $(\mathrm{p}=0.37)$. The PS in controls remained unchanged on repeat testing after an average 19.1 days $(48.4 \pm 17.1, \mathrm{p}=0.61)$.

Using GEE we found that time of measurement was a significant predictor of phase shift in the affected hemisphere $\left(\chi^{2}=6.4, p=0.04\right.$ and $\chi^{2}=11, p=0.004$, respectively). Using the last recording as the reference, the mean PS in the affected hemisphere was $-23.54(\mathrm{CI}-44.1,-3)$ degrees lower on recording one $(\mathrm{p}=0.025)$, and $-31.6(\mathrm{CI}$ $-56.1,-7.1)$ degrees lower on recording two $(\mathrm{p}<0.011)$. Changes in the unaffected hemisphere over time were non-significant. Figure 2 shows error bars of the phase shift.

\section{Discussion}

This study suggests that DCA is impaired in the affected hemisphere early after moderate-to-severe anterior circulation ischemic stroke. In our cohort autoregulation remained impaired throughout the first week and 

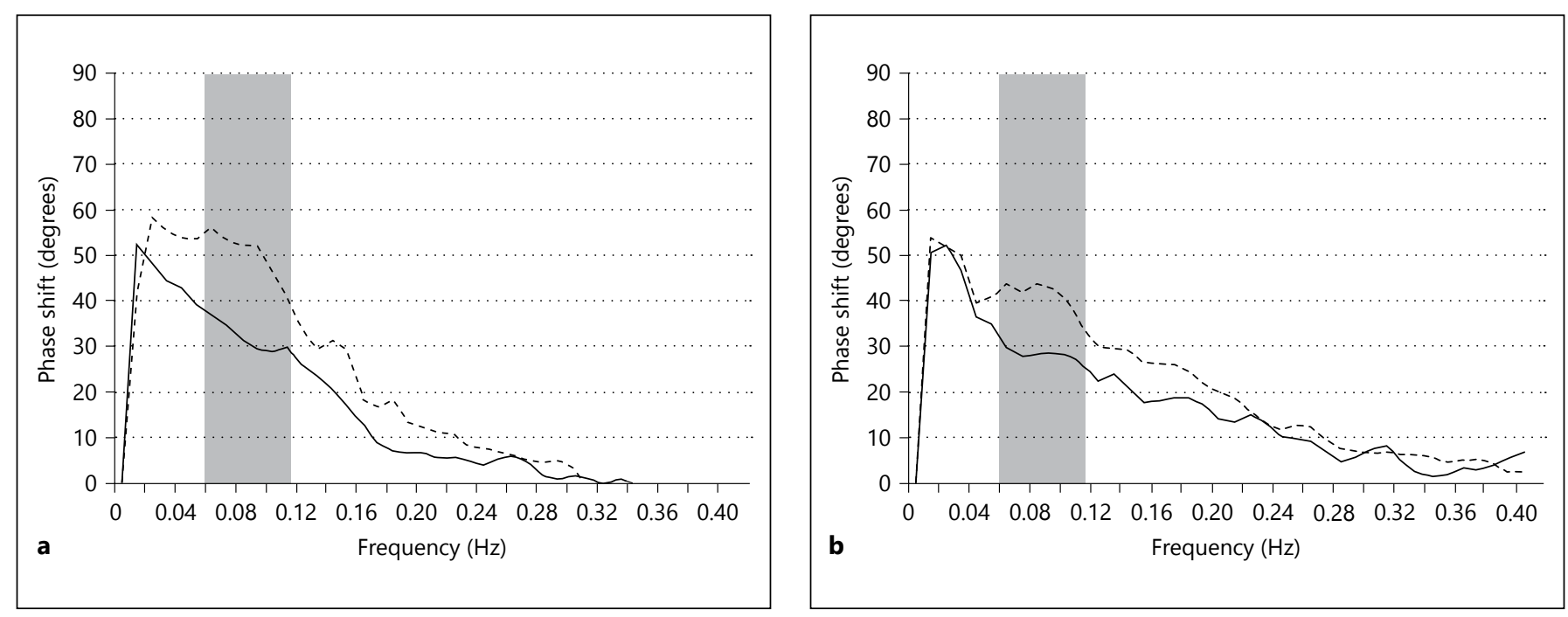

Fig. 1. a-c Average phase shift between mean ABP and CBFV on measurement 1 (a), measurement 2 (b) and measurement 3 (c) for the affected hemisphere (dashed line) and unaffected hemisphere (solid line). Gray bar represent frequency range of interest (0.06$0.12 \mathrm{~Hz}$ ).

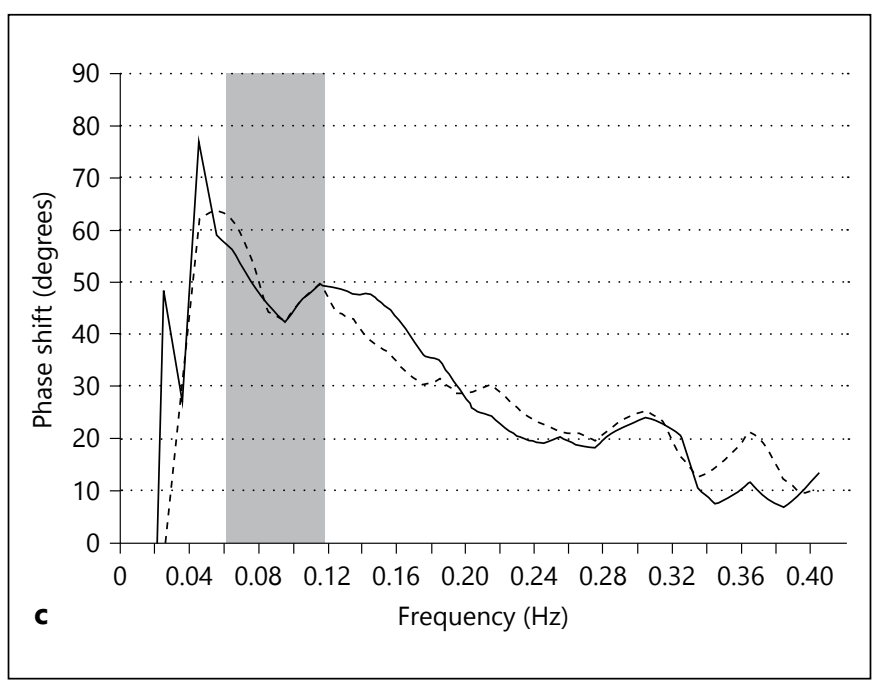

Table 2. Hemodynamic parameters

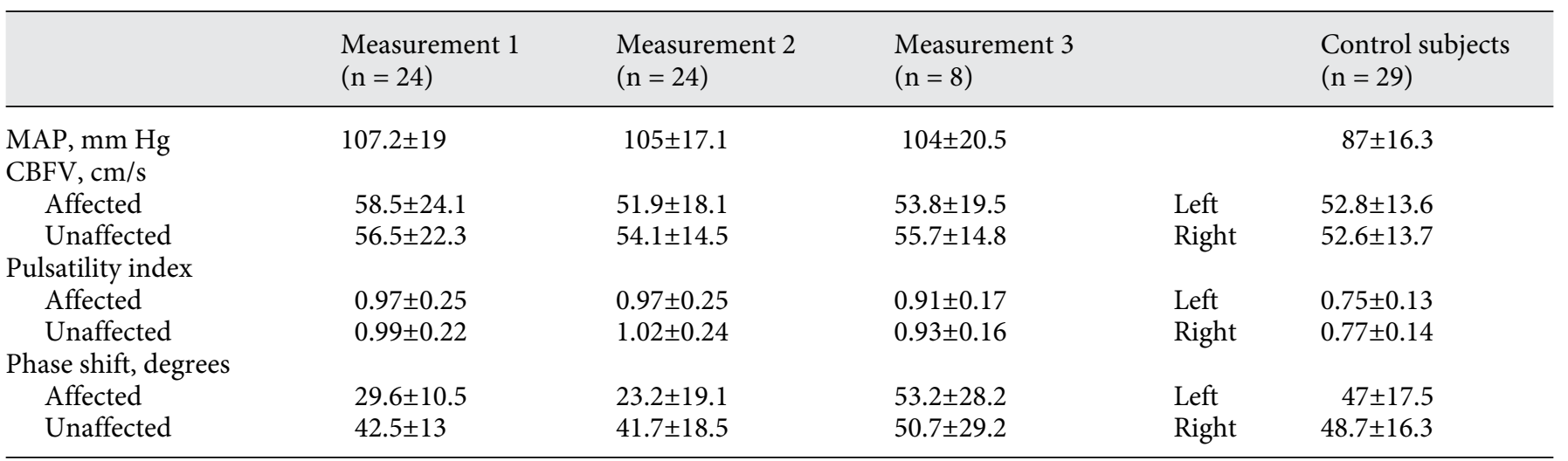

Values represent mean $\pm \mathrm{SD}$. 


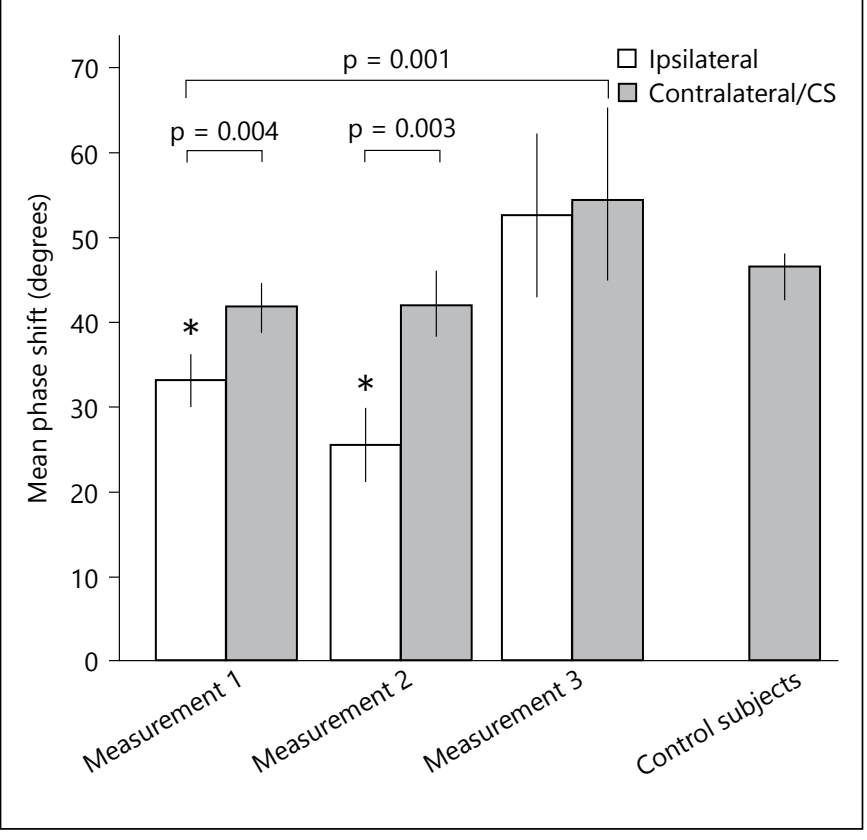

Fig. 2. Course of DCA in patients with acute MCA Stroke compared to control subjects for phase shift. Bars represent means, whiskers denote standard error. ${ }^{*}$ Indicates significance $(\mathrm{p}<0.05)$ for comparison with control subjects.

then normalized by day ten. Previous studies evaluating the early course of cerebral autoregulation after stroke reported similar results $[3-6,20]$. Reinhard et al. reported increasingly disturbed autoregulation in the affected hemisphere within the first days after large anterior circulation ischemic stroke. Their patient selection and methods to measure DCA are comparable to the ones used in our study, and the absolute phase values found in our study are similar to those they reported $[3,4]$. However, they focused on the early phase after ischemic stroke, and did not follow their patients beyond the first week. Our study is the first to demonstrate the recovery of autoregulation after acute ischemic stroke.

The pathophysiology for impaired autoregulation is not known. It is believed that tissue lactic acidosis leads to local vasoparalysis in the infarcted area and immediate peri-infarct tissue, inhibiting normal autoregulatory function. Invasive measurements with microdialysis in patients with malignant MCA infarction have shown a close correlation between worsening lactate/pyruvate ratio and impaired autoregulation in the peri-infarct tissue, suggesting that lactate and extracellular fluid spreading along the white matter tracts to the peri-infarct tissue may impair autoregulation in this compartment [21]. This process seems to persist throughout the early post-stroke period, possibly accounting for worsening autoregulation in the first few days after stroke. In this time period, regions of suboptimal flow, which were present within the first hours after stroke, have resolved into an ischemic state with low flow in the infarct, surrounded by histologically normal peri-infarct tissue with normal resting flow. Animal models have confirmed that this peri-infarct tissue nonetheless still exhibits impaired autoregulatory function, at least at $24 \mathrm{~h}$ after stroke [22].

DCA is probably normal in the chronic phase after ischemic stroke, although data is limited and methods to assess DCA vary considerably [23-25]. There is even less information at what time recovery of autoregulation occurs. In our study, autoregulation had recovered by day ten after acute anterior circulation ischemic stroke. This has important implications for blood pressure management in the acute stroke period. Instability in blood pressure is very common after ischemic stroke [26] and its management remains unclear. During autoregulatory failure, the brain tissue is particularly vulnerable to blood pressure changes, as they have direct effects on cerebral blood flow. Our data suggests that the period of autoregulatory impairment lasts at least 1 week. During that time careful blood pressure monitoring and tight external regulation of blood pressure may be critical to avoid secondary worsening.

Our findings contrast Dawson et al. who reported persistence of DCA impairment at 7-14 days [27]. However, they used the autoregulatory index (ARI) instead of phase shift to assess DCA. The ARI compares the CBFV response after a rapid step-like decrease in blood pressure with 10 theoretical flow-velocity curves $[1,15]$. A single episode of induced hypotension (e.g., thigh cuff deflation) may not measure the same as the response to spontaneous oscillatory changes in blood pressure. The two measurement methods may represent two distinct processes and their recovery after stroke may be dissociated.

In addition, stroke size and etiology may have played a role. Our study population was very uniform and only comprised of patients with partial anterior circulation stroke. The Dawson study also utilized a more heterogeneous study group, including patients with total anterior circulation, posterior circulation and lacunar stroke. $\mathrm{Pa}$ tients with lacunar stroke in particular may have more persistent impairment of DCA, possibly reflecting preexisting endothelial dysfunction due to hypertensive smallvessel disease or metabolic syndrome. This underlying dysfunction may be exacerbated during the acute phase response of an ischemic stroke, when inflammatory 
changes also affect the cerebral vasculature [4, 28, 29]. This small vessel hypothesis is consistent with the finding of bilateral autoregulatory impairment in patients after lacunar stroke [5]. Thus, the lack of autoregulatory improvement in the Dawson study may be explained at least in part by the inclusion of patients with lacunar stroke who have more persistent impairment of DCA, thereby masking an improvement in autoregulatory function in patients with other stroke subtypes.

This study has limitations including its relatively small sample size in particular at the third measurement, operator-dependence of transcranial Doppler and limited temporal windows for TCD measurement in some patients. In addition, we did not control for white matter hyperintensity or lacunar stroke burden, although the asymmetry between the affected and unaffected hemi- spheres suggests that the acute infarct itself produced the effect we observed.

In summary, dysautoregulation appears to take place early after moderate-to-severe ischemic stroke and normalize in the subacute period by day ten. Our findings have important implications for blood pressure management in the acute phase of ischemic stroke. Prospective validation of noninvasive measures of cerebral autoregulation should be confirmed in a larger study before individualized blood pressure can be managed routinely based on autoregulatory function.

\section{Disclosure Statement}

None.

\section{References}

1 Tiecks FP, Lam AM, Aaslid R, Newell DW: Comparison of static and dynamic cerebral autoregulation measurements. Stroke 1995; 26:1014-1019.

2 Aaslid R, Lindegaard KF, Sorteberg W, Nornes H: Cerebral autoregulation dynamics in humans. Stroke 1989;20:45-52.

-3 Reinhard M, Wihler C, Roth M, Harloff A, Niesen WD, Timmer J, et al: Cerebral autoregulation dynamics in acute ischemic stroke after rtPA thrombolysis. Cerebrovasc Dis 2008;26:147-155.

-4 Reinhard M, Rutsch S, Lambeck J, Wihler C, Czosnyka M, Weiller C, et al: Dynamic cerebral autoregulation associates with infarct size and outcome after ischemic stroke. Acta Neurol Scand 2012;125:156-162.

5 Immink RV, van Montfrans GA, Stam J, Karemaker JM, Diamant M, van Lieshout JJ: Dynamic cerebral autoregulation in acute lacunar and middle cerebral artery territory ischemic stroke. Stroke 2005;36:2595-2600.

-6 Atkins ER, Brodie FG, Rafelt SE, Panerai RB, Robinson TG: Dynamic cerebral autoregulation is compromised acutely following mild ischaemic stroke but not transient ischaemic attack. Cerebrovasc Dis 2010;29:228-235.

7 Semplicini A, Maresca A, Boscolo G, Sartori M, Rocchi R, Giantin V, et al: Hypertension in acute ischemic stroke: a compensatory mechanism or an additional damaging factor? Arch Intern Med 2003;163:211-216.

-8 Qureshi AI, Ezzeddine MA, Nasar A, Suri MF, Kirmani JF, Hussein HM, et al: Prevalence of elevated blood pressure in 563,704 adult patients with stroke presenting to the ED in the United States. Am J Emerg Med 2007;25:32-38.

9 Castillo J, Leira R, García MM, Serena J, Blanco M, Dávalos A: Blood pressure decrease during the acute phase of ischemic stroke is associated with brain injury and poor stroke outcome. Stroke 2004;35:520-526.

10 Aslanyan S, Fazekas F, Weir CJ, Horner S, Lees KR; GAIN International Steering Committee and Investigators: Effect of blood pressure during the acute period of ischemic stroke on stroke outcome: a tertiary analysis of the GAIN International Trial. Stroke 2003;34: 2420-2425.

-11 Sandset EC, Murray GD, Bath PM, Kjeldsen SE, Berge E; Scandinavian Candesartan Acute Stroke Trial (SCAST) Study Group: Relation between change in blood pressure in acute stroke and risk of early adverse events and poor outcome. Stroke 2012;43:2108-2114.

-12 Adams HP Jr, del Zoppo G, Alberts MJ, Bhatt DL, Brass L, Furlan A, et al: Guidelines for the early management of adults with ischemic stroke: a guideline from the American Heart Association/American Stroke Association Stroke Council, Clinical Cardiology Council, Cardiovascular Radiology and Intervention Council, and the Atherosclerotic Peripheral Vascular Disease and Quality of Care Outcomes in Research Interdisciplinary Working Groups: The American Academy of Neurology affirms the value of this guideline as an educational tool for neurologists. Circulation 2007;115:e478-e534.

13 Zhang R, Zuckerman JH, Giller CA, Levine $\mathrm{BD}$ : Transfer function analysis of dynamic cerebral autoregulation in humans. Am J Physiol 1998;274:H233-H241.

14 Reinhard M, Muller T, Guschlbauer B, Timmer J, Hetzel A: Transfer function analysis for clinical evaluation of dynamic cerebral autoregulation - a comparison between spontaneous and respiratory-induced oscillations. Physiol Meas 2003;24:27-43.
15 van Beek $\mathrm{AH}$, Claassen JA, Rikkert MG, Jansen RW: Cerebral autoregulation: an overview of current concepts and methodology with special focus on the elderly. J Cereb Blood Flow Metab 2008;28:10711085.

16 Welch P: The use of fast Fourier transform for the estimation of power spectra: a method based on time averaging over short, modified periodograms. IEEE Transactions on Audio and Electroacoustics, 1967, vol 15, pp 70-73.

17 Ortega-Gutierrez S, Petersen N, Masurkar A, Reccius A, Huang A, Li M, et al: Reliability, asymmetry, and age influence on dynamic cerebral autoregulation measured by spontaneous fluctuations of blood pressure and cerebral blood flow velocities in healthy individuals. J Neuroimaging 2014;24:379386.

18 Gommer ED, Shijaku E, Mess WH, Reulen JP: Dynamic cerebral autoregulation: different signal processing methods without influence on results and reproducibility. Med Biol Eng Comput 2010;48:1243-1250.

19 Kuo TB, Chern CM, Yang CC, Hsu HY, Wong WJ, Sheng WY, et al: Mechanisms underlying phase lag between systemic arterial blood pressure and cerebral blood flow velocity. Cerebrovasc Dis 2003;16:402-409.

20 Reinhard M: Dynamic cerebral autoregulation in acute ischemic stroke assessed from spontaneous blood pressure fluctuations. Stroke 2005;36:1684-1689.

21 Dohmen C, Bosche B, Graf R, Reithmeier T, Ernestus RI, Brinker G, et al: Identification and clinical impact of impaired cerebrovascular autoregulation in patients with malignant middle cerebral artery infarction. Stroke 2007;38:56-61.
Transient Impairment of DCA after Ischemic Stroke
Cerebrovasc Dis 2015;39:144-150 DOI: 10.1159/000368595 
22 MacGregor DG, Carswell HV, Graham DI, McCulloch J, Macrae IM: Impaired cerebral autoregulation $24 \mathrm{~h}$ after induction of transient unilateral focal ischaemia in the rat. Eur J Neurosci 2000;12:58-66.

23 Kwan J, Lunt M, Jenkinson D: Assessing dynamic cerebral autoregulation after stroke using a novel technique of combining transcranial Doppler ultrasonography and rhythmic handgrip. Blood Press Monit 2004;9:3-8.

-24 Novak V, Chowdhary A, Farrar B, Nagaraja H, Braun J, Kanard R, et al: Altered cerebral vasoregulation in hypertension and stroke. Neurology 2003;60:1657-1663.
25 Gommer ED, Staals J, van Oostenbrugge RJ, Lodder J, Mess WH, Reulen JP: Dynamic cerebral autoregulation and cerebrovascular reactivity: a comparative study in lacunar infarct patients. Physiol Meas 2008;29:1293-1303.

26 Carlberg B, Asplund K, Hägg E: The prognostic value of admission blood pressure in patients with acute stroke. Stroke 1993;24:13721375.
Dawson SL, Panerai RB, Potter JF: Seria changes in static and dynamic cerebral autoregulation after acute ischaemic stroke. Cerebrovasc Dis 2003;16:69-75.

28 Stevenson SF, Doubal FN, Shuler K, Wardlaw JM: A systematic review of dynamic cerebral and peripheral endothelial function in lacunar stroke versus controls. Stroke 2010; 41:e434-e442.

29 Giannopoulos S, Boden-Albala B, Choi JH, Carrera E, Doyle M, Perez T, et al: Metabolic syndrome and cerebral vasomotor reactivity. Eur J Neurol 2010;17:1457-1462. 